\title{
Hepatitis C-associated late-onset schizophrenia: a nationwide, population-based cohort study
}

\author{
Jur-Shan Cheng, PhD; Jing-Hong Hu, MD; Ming-Yu Chang, MD; Ming-Shyan Lin, MD; \\ Hsin-Ping Ku, MS; Rong-Nan Chien, MD; Ming-Ling Chang, MD, PhD
}

\begin{abstract}
Background: Whether infection with the hepatitis $\mathrm{C}$ virus $(\mathrm{HCV})$ causes schizophrenia - and whether the associated risk reverses after anti-HCV therapy - is unknown; we aimed to investigate these topics. Methods: We conducted a nationwide, population-based cohort study using the Taiwan National Health Insurance Research Database (TNHIRD). A diagnosis of schizophrenia was based on criteria from the International Classification of Diseases, 9th revision (295.xx). Results: From 2003 to 2012, from a total population of 19298735, we enrolled 3 propensity-score-matched cohorts (1:2:2): HCV-treated (8931 HCV-infected patients who had received interferon-based therapy for $\geq 6$ months); HCV-untreated (17862); and HCV-uninfected (17862) from the TNHIRD. Of the total sample (44655), $82.81 \%$ (36980) were 40 years of age or older. Of the 3 cohorts, the HCV-untreated group had the highest 9 -year cumulative incidence of schizophrenia $(0.870 \%, 95 \%$ confidence interval $[\mathrm{Cl}] 0.556 \%-1.311 \% ; p<0.001)$; the $\mathrm{HCV}$-treated $(0.251 \%, 95 \% \mathrm{Cl}$ $0.091 \%-0.599 \%)$ and HCV-uninfected $(0.118 \%, 95 \% \mathrm{Cl} 0.062 \%-0.213 \%)$ cohorts showed similar cumulative incidence of schizophrenia $(p=0.33$ ). Multivariate Cox analyses showed that HCV positivity (hazard ratio [HR] $3.469,95 \% \mathrm{Cl} 2.168-5.551$ ) was independently associated with the development of schizophrenia. The HCV-untreated cohort also had the highest cumulative incidence of overall mortality (20.799\%, 95\% Cl 18.739\%-22.936\%; $p<0.001)$; the HCV-treated (12.518\%, 95\% Cl 8.707\%-17.052\%) and HCVuninfected (6.707\%, $95 \% \mathrm{Cl} 5.533 \%-8.026 \%)$ cohorts showed similar cumulative incidence of mortality $(p=0.12)$. Limitations: We were unable to determine the precise mechanism of the increased risk of schizophrenia in patients with HCV infection. Conclusion: In a population-based cohort (most aged $\geq 40$ years), HCV positivity was a potential risk factor for the development of schizophrenia; the HCV-associated risk of schizophrenia might be reversed by interferon-based antiviral therapy.
\end{abstract}

\section{Introduction}

Hepatitis $\mathrm{C}$ virus (HCV) is a human pathogen responsible for acute and chronic liver disease that infects an estimated 150 million people worldwide, ${ }^{1}$ with an estimated global prevalence of $2.8 \% .^{2} \mathrm{HCV}$ infection is considered a systemic disease. In addition to causing hepatic complications such as steatosis, cirrhosis and hepatocellular carcinoma, HCV also causes extrahepatic complications, including mixed cryoglobulinemia, renal diseases, ${ }^{3}$ hypolipidemia, diabetes mellitus, obesity, cardiovascular disease ${ }^{1}$ and neurologic and psychiatric events. ${ }^{4}$ Neuropsychiatric disorders have been reported in up to $50 \%$ of patients with chronic HCV infection, independent of the severity of the underlying liver disease. ${ }^{4}$

Schizophrenia is a debilitating psychiatric illness that affects approximately $1.1 \%$ of the world's population, amounting to approximately 51 million people. ${ }^{5}$ The lifetime prevalence of schizophrenia is approximately $1 \%$, and it commonly follows a chronic course, with onset in late adolescence. ${ }^{6}$ Schizophrenia contributes to a substantial chronic medical burden and a lifespan that is approximately 15 years shorter than the general population. ${ }^{7}$ The diverse clinical manifestations of schizophrenia include cognitive, emotional and behavioural abnormalities. ${ }^{6}$ Current treatments for schizophrenia target mainly psychotic symptoms, do little to ameliorate social or cognitive deficits, and come with adverse effects such as weight gain and diabetes; as well, $30 \%$ of people do not respond to them. ${ }^{8}$

The relationship between schizophrenia and HCV infection has been investigated, but findings have been conflicting. For example, a US study by the Veterans Integrated Services Network showed that patients with schizophrenia had a higher prevalence of HCV infection than the general population (16.5\% v. $1.9 \%) .{ }^{9}$ Similarly, reported HCV infection rates in patients with schizophrenia are $6.2 \%$ in $\operatorname{Japan}^{10}$

Correspondence to: M. L. Chang, Liver Research Center, Division of Hepatology, Department of Gastroenterology and Hepatology, Chang Gung Memorial Hospital, No. 5 Fu Hsing Street, Kuei Shan, Taoyuan, Taiwan; mlchang8210@ gmail.com

Submitted Nov. 1, 2020; Revised Jul. 7, 2021; Accepted Jul. 26, 2021

Cite as: J Psychiatry Neurosci 2021 November 2;46(6). doi: 10.1503/jpn.200154 
and $7.1 \%$ and $8.2 \%$ in the United States, ${ }^{11,12}$ higher than the estimated global HCV prevalence $(2.8 \%){ }^{2}$ In contrast, a study showed that the seroprevalence of the HCV antibody was $1.9 \%$ in institutionalized patients with chronic schizophrenia, ${ }^{13}$ and another case-control study showed that the concurrent $\mathrm{HCV}$ infection rate was $2.1 \%$ in patients with schizophrenia ${ }^{14}$ - both similar to the prevalence of $\mathrm{HCV}$ in the general population. The situation is complicated further when the therapeutic effects of anti-HCV treatment are considered. The combination of pegylated interferon (PEG-IFN) and ribavirin has provided a "cure" for a considerable proportion of patients with chronic HCV infection, particularly patients with the favourable interferon $\lambda 3$ (IFNL3) genotype. ${ }^{15}$ Cure rates have been further improved by replacing interferon-based therapy with potent direct-acting antiviral agents (DAAs). ${ }^{1}$ The rate of sustained virological response (SVR) to DAAs in HCV-infected patients is approximately $100 \% .{ }^{16}$ Still, although most HCV infections are currently curable with potent DAAs, not all HCV-associated complications are reversed after viral clearance. ${ }^{1,17}$ Thus, whether $\mathrm{HCV}$ infection accelerates the risk of developing schizophrenia and whether the associated risk is reversed after anti-HCV therapy remain crucial issues for public health in an era when HCV infection can be cured using DAAs.

We conducted a nationwide population-based cohort study to investigate the effects of HCV infection on the development of schizophrenia in Taiwan, a country where HCV infection is rampant. ${ }^{18}$ We investigated the impact of HCV infection on schizophrenia by comparing the cumulative incidence of schizophrenia and of overall mortality among HCVinfected people with and without anti-HCV therapy, and among people without $\mathrm{HCV}$ infection.

\section{Methods}

\section{Samples and measurements}

This population-based retrospective cohort study used national-level data from the Taiwan National Health Insurance Research Database (TNHIRD), including the National Health Insurance (NHI) administrative database, the cancer registry database and the death registry database. The mandatory, single-payer NHI program provides comprehensive coverage of ambulatory care, laboratory tests, hospital services and prescription drugs. More than $99 \%$ of the population is enrolled in the program, and approximately $92 \%$ of health care organizations are contracted with the NHI administration. We did not enrol patients who had schizophrenia before they started anti-HCV treatment in the HCVtreated cohort, or before baseline in the other cohorts. Because Taiwan is a hyperendemic area for hepatitis B virus infection, which profoundly affects $\mathrm{HCV}$ viral load and biases the phenotype of HCV infection, ${ }^{19}$ we excluded patients who were infected with hepatitis $B$ virus during the observation period (2003-2012). We also excluded patients with cirrhosis-related complications such as hepatoencephalopathy, esophageal varices, ascites or hepatorenal syndrome to avoid interference from complications or from as- sociated treatments. In particular, given that some infections such as toxoplasmosis, ${ }^{20,21}$ Chlamydophila pneumoniae, ${ }^{22}$ lymphocytic choriomeningitis virus ${ }^{23}$ and herpes simplex virus type $1^{24}$ have been reported to affect the development of schizophrenia, we excluded patients with these infections.

The HCV-treated cohort included patients who had an HCV RNA test and received ribavirin and PEG-IFN between 2003 and 2012. The date of their first HCV test was assumed to be the index date of their diagnosis. Baseline for the HCVtreated cohort was 6 months after they had completed combination therapy, which is the time to ensure SVR. Only those who had undergone anti-HCV therapy for 6 months or more were enrolled. The HCV-untreated cohort included patients who had an HCV test (HCV antibody or HCV RNA test; their first $\mathrm{HCV}$ test was the index date); were diagnosed with hepatitis C (International Classification of Diseases, 9th revision, clinical modification [ICD-9-CM] codes 070.41, 070.44, 070.51, $070.54,070.70,070.71$ or V02.62); were prescribed a hepatoprotective agent (silymarin, liver hydrolysate, choline bitartrate or ursodeoxycholic acid); and did not receive any antiHCV therapy (ribavirin or PEG-IFN). The HCV-uninfected cohort included those who had no diagnosis of HCV and no $\mathrm{HCV}$ test, and who received no hepatoprotective agents or anti-HCV therapy. Only those without schizophrenia-related $\mathrm{NHI}$ claims at least 2 years before baseline were included in the 3 cohorts.

We matched the HCV-treated cohort with the HCVuntreated and HCV-uninfected cohorts (1:2:2) using a propensity score-matching method that indicated the probability of receiving combination therapy and was estimated using a logistic model. Covariates in the model included age group (20-39, 40-49, 50-59, $\geq 60$ years), sex (male, female), NHI registration location (urban, township, rural), Charlson Comorbidity Index score $(0,1, \geq 2)^{25}$ and the year of the index date (2003-2006, 2007-2009, 2010-2012). We used this method to ensure that the HCV-treated cohort and their selected counterparts were comparable in terms of observed characteristics. We assigned the baseline for the $\mathrm{HCV}$-untreated and $\mathrm{HCV}$-uninfected cohorts according to the period from the index date to baseline $(6$ months after treatment completion) for their matched counterparts. The index date for those in the HCV-uninfected cohort was the date of a physician visit randomly selected from their claims database. The matching process for the 3 cohorts is shown in Appendix 1, Figure S1, available at jpn.ca. Outcomes were the development of schizophrenia or mortality. Schizophrenia was defined as follows: at least 1 hospitalization with a schizophrenia diagnosis (ICD-9-CM code 295.xx); at least 2 outpatient visits with a schizophrenia diagnosis; or at least 1 outpatient visit with a schizophrenia diagnosis and at least 1 antipsychotic prescription within 1 year. Patients were followed until the date of an event (schizophrenia), death or the end of follow-up (December 31, 2012), whichever came first. For the HCV-treated group, we recorded only schizophrenia diagnoses or death that occurred more than 6 months after the completion of anti$\mathrm{HCV}$ therapy. Dates of death were taken from the death registry database. 


\section{Statistical analysis}

We performed all statistical analyses using SAS statistical software (version 9.4; SAS Institute Inc.). We estimated cumulative incidences of outcomes and compared them using the modified Kaplan-Meier method and the Gray method; death was a competing risk event. ${ }^{26}$ We used Cox proportional hazards models, taking competing mortality into account, to estimate adjusted hazard ratios for developing schizophrenia - adjusting for age; sex; the interaction of age and sex; NHI registration location; Charlson Comorbidity Index score; year of index date; and the baseline presence of liver cirrhosis, chronic obstructive pulmonary disease, end-stage renal disease, diabetes mellitus, hypertension, dyslipidemia, cardiovascular events (including percutaneous coronary intervention, coronary artery bypass graft, myocardial infarction, heart failure, cardiogenic shock and peripheral vascular disease) and stroke. We checked and confirmed proportionality assumptions using time-dependent explanatory variables. We checked for multicollinearity and excluded variables that were highly correlated with other variables. We conducted a post hoc power analysis to assess the powers of the tests used in this study. Statistical significance was set at $5 \%$ based on 2 -tailed tests of the null hypothesis.

\section{Institutional review board}

The study protocol conformed to the ethical guidelines of the 1975 Declaration of Helsinki and was approved by the local institutional review board.

\section{Results}

\section{Baseline characteristics}

From a total of 19298735 patients registered in the TNHIRD database between January 1, 2003, and December 31, 2012, 112680 patients with HCV infection and 11686609 patients without $\mathrm{HCV}$ infection were eligible for the study. We enrolled patients in 1 of 3 cohorts: HCV-treated $(n=8931)$, HCV-untreated $(n=17862)$ and HCV-uninfected $(n=17862$; Figure 1). The 3 cohorts were matched using propensity scores and did not differ in terms of demographic factors, $\mathrm{NHI}$ registration location, Charlson Comorbidity Index score or index year - the covariates used to calculate propensity scores in the models; baseline comorbidities differed. Of the total sample $(n=44655), 82.81 \%(n=36980)$ were age 40 years or older (Table 1). Compared to the HCV-untreated cohort, the HCV-treated cohort had higher rates of baseline cirrhosis but lower rates of end-stage renal disease, diabetes mellitus, hypertension, dyslipidemia, cardiovascular events and stroke. Compared to the HCV-uninfected cohort, the HCV-treated cohort had higher rates of baseline cirrhosis, chronic obstructive pulmonary disease and end-stage renal disease, but lower rates of dyslipidemia and stroke. Compared to the HCV-uninfected cohort, the HCV-untreated cohort had higher rates of all baseline comorbidities except stroke. To delineate which complications were associated with $\mathrm{HCV}$ infection, we compared baseline factors between an HCV-infected cohort (a combination of the HCV-treated and HCV-untreated cohorts) and the HCV-uninfected cohort. The HCV-infected cohort had higher rates of all baseline comorbidities, except for dyslipidemia and stroke (Appendix 1, Table S1).

\section{Cumulative incidence of schizophrenia and associated risk factors}

We followed the 3 cohorts (HCV-treated, HCV-untreated and HCV-uninfected until death, for a duration of up to 9.5 years). The results of the post hoc power analysis for the various comparisons were greater than 0.8 (Appendix 1, Table S2). Of the 3 cohorts, the HCV-untreated cohort had the highest cumulative incidence of schizophrenia (Figure 2, Table 2). However, we observed no difference in cumulative incidence of schizophrenia between the HCV-treated and HCV-uninfected cohorts. Multivariate analysis of the 3 cohorts showed that the HCV-untreated cohort had a higher risk of developing schizophrenia than the HCV-treated and $\mathrm{HCV}$-uninfected cohorts, and that patients with baseline stroke had borderline higher risk than those without baseline stroke (Appendix 1, Figure S2).

Because the HCV-treated and HCV-uninfected cohorts showed similar risks of developing schizophrenia, we combined these cohorts into an HCV-negative cohort to explore the effect of $\mathrm{HCV}$ on the development of schizophrenia. Compared to the HCV-negative cohort, the HCV-positive (i.e., HCV-untreated) cohort had a higher risk of incident schizophrenia $(p<0.001$; Appendix 1, Table S3). Multivariate analyses of these 2 cohorts showed that HCV positivity (hazard ratio $3.469,95 \%$ confidence interval [CI] 2.168-5.551) was an independent risk factor for the development of schizophrenia (Figure 3).

\section{Cumulative incidence of mortality}

Of the 3 cohorts, the HCV-untreated cohort had the highest cumulative incidence of overall mortality at 9.5 years (20.799\%, 95\% CI 18.739\%-22.936\%; $p<0.001)$; the HCVtreated $(12.518 \%, 95 \%$ CI $8.707 \%-17.052 \%)$ and HCVuninfected cohorts $(6.707 \%, 95 \%$ CI $5.533 \%-8.026 \%)$ had similar mortality rates ( $p=0.12$; Appendix 1 , Table S4).

\section{Discussion}

The differences in baseline comorbidities between the HCVtreated and HCV-untreated cohorts coincided with the fact that only patients with significant hepatic fibrosis were reimbursed for anti-HCV therapy, and those with severe comorbidities were ineligible for interferon-based therapy. The differences in comorbidities between the HCV-infected and HCV-uninfected cohorts reflected the fact that HCV is associated with cardiometabolic complications and that people infected with $\mathrm{HCV}$ are less likely to have dyslipidemia. ${ }^{1}$ Thus, our baseline comparisons of the 3 cohorts supported the reliability of the results from the TNHIRD. 
152851 patients had an HCV diagnosis, an HCV test and received hepatoprotective agents (ribavirin or PEG-IFN) between 2003 and 2012
19145884 people had no HCV diagnosis, no HCV test and no HCV-related pharmacotherapy between 2003 and 2012
40171 patients excluded for:

- Age < 20 yr or $>70 \mathrm{yr}$

- History of HBV infection, schizophrenia, esophageal or gastric varices, hepatic encephalopathy, hepatorenal syndrome or ascites
7459275 excluded for:

- Age < 20 yr or $>70 \mathrm{yr}$

- History of HBV infection, schizophrenia, esophageal or gastric varices, hepatic encephalopathy, hepatorenal syndrome, ascites, Toxoplasma gondii, Chlamydophila pneumoniae, lymphocytic choriomeningitis or HSV1 brain infection

14027 excluded for:

- No HCV RNA test

- $<24$ wk of therapy

- Event or mortality occurred $<24$ weeks after end of therapy

- Toxoplasma gondii, Chlamydophila pneumoniae, lymphocytic choriomeningitis or HSV1 brain infection

9114 patients received ribavirin and PEG-IFN therapy
11686609 eligible people

89539 patients did not receive ribavirin and PEG-IFN therapy

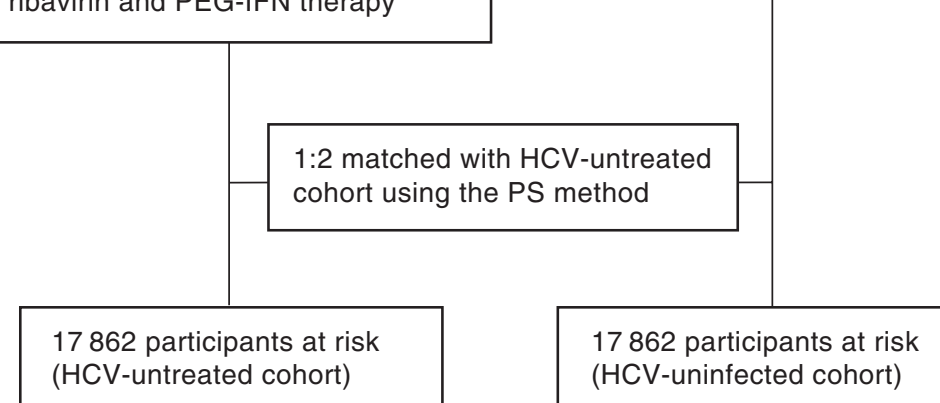

17862 participants at risk (HCV-uninfected cohort)
8931 participants at risk (HCV-treated cohort)

Figure 1: Flow chart of Taiwan National Health Insurance Research Database study sample selection. HBV = hepatitis B virus; HCV = hepatitis C virus; HSV = herpes simplex virus; PEG-IFN: pegylated interferon; PS = propensity score.

Although high HCV infection rates have been noted in patients with schizophrenia, the data are conflicting, ${ }^{9-14}$ and high comorbidity with intravenous substance use ${ }^{11}$ or old age $\mathrm{e}^{10}$ in patients with schizophrenia has been thought to account for the high $\mathrm{HCV}$ prevalence in this population.
The present study clearly demonstrated that HCV infection increased the risk of incident schizophrenia: the HCVuntreated cohort had the highest cumulative incidence of schizophrenia of the 3 cohorts, and multivariate analyses showed that $\mathrm{HCV}$ infection was positively associated with 


\begin{tabular}{|c|c|c|c|c|c|c|}
\hline \multirow[b]{2}{*}{ Characteristic } & \multirow[b]{2}{*}{ HCV-treated } & \multirow[b]{2}{*}{ HCV-untreated } & \multirow[b]{2}{*}{ HCV-uninfected } & \multicolumn{3}{|c|}{$p$ value } \\
\hline & & & & $\begin{array}{l}\text { HCV-treated } \\
\text { v. HCV- } \\
\text { untreated }\end{array}$ & $\begin{array}{l}\text { HCV-treated } \\
\text { v. HCV- } \\
\text { uninfected }\end{array}$ & $\begin{array}{c}\text { HCV-untreated } \\
\text { v. HCV- } \\
\text { uninfected }\end{array}$ \\
\hline Participants & 8931 & 17862 & 17862 & & & \\
\hline \multicolumn{7}{|l|}{ Sex } \\
\hline Male & $4810(53.86)$ & $9620(53.86)$ & $9620(53.86)$ & $>0.99$ & $>0.99$ & $>0.99$ \\
\hline Female & $4121(46.14)$ & $8242(46.14)$ & $8242(46.14)$ & & & \\
\hline Mean age, yr & $50.22 \pm 10.61$ & $50.33 \pm 11.11$ & $49.72 \pm 11.72$ & & & \\
\hline \multicolumn{7}{|l|}{ Age range, yr } \\
\hline 20-39 & $1535(17.19)$ & $3070(17.19)$ & $3070(17.19)$ & $>0.99$ & $>0.99$ & $>0.99$ \\
\hline $40-49$ & $2473(27.69)$ & $4946(27.69)$ & $4946(27.69)$ & & & \\
\hline $50-59$ & $3242(36.30)$ & $6484(36.30)$ & $6484(36.30)$ & & & \\
\hline$\geq 60$ & $1681(18.82)$ & $3362(18.82)$ & $3362(18.82)$ & & & \\
\hline \multicolumn{7}{|l|}{$\mathrm{NHI}$ registration location } \\
\hline Urban & $2169(24.29)$ & $4338(24.29)$ & $4338(24.29)$ & $>0.99$ & $>0.99$ & $>0.99$ \\
\hline Township & $2755(30.85)$ & $5510(30.85)$ & $5510(30.85)$ & & & \\
\hline Rural & $4007(44.87)$ & $8014(44.87)$ & $8014(44.87)$ & & & \\
\hline \multicolumn{7}{|l|}{ Charlson Comorbidity Index score } \\
\hline 0 & $4244(47.52)$ & $8488(47.52)$ & $8488(47.52)$ & $>0.99$ & $>0.99$ & $>0.99$ \\
\hline 1 & $2992(33.50)$ & $5984(33.50)$ & $5984(33.50)$ & & & \\
\hline$\geq 2$ & 1695 (18.98) & $3390(18.98)$ & $3390(18.98)$ & & & \\
\hline \multicolumn{7}{|l|}{ Index year } \\
\hline 2003-2006 & $4348(48.68)$ & 8696 (48.68) & $8696(48.68)$ & $>0.99$ & $>0.99$ & $>0.99$ \\
\hline 2007-2009 & 2775 (31.07) & $5550(31.07)$ & $5550(31.07)$ & & & \\
\hline 2010-2012 & $1808(20.24)$ & $3616(20.24)$ & $3616(20.24)$ & & & \\
\hline \multicolumn{7}{|l|}{ Baseline risk factor } \\
\hline Liver cirrhosis & $965(10.81)$ & $1071(6.00)$ & $7(0.04)$ & $<0.001$ & $<0.001$ & $<0.001$ \\
\hline Chronic obstructive pulmonary disease & $1033(11.57)$ & $2094(11.72)$ & $1733(9.70)$ & 0.70 & $<0.001$ & $<0.001$ \\
\hline End-stage renal disease & $60(0.67)$ & $499(2.79)$ & $41(0.23)$ & $<0.001$ & $<0.001$ & $<0.001$ \\
\hline Diabetes mellitus & 1673 (18.73) & 4071 (22.79) & 3254 (18.22) & $<0.001$ & 0.30 & $<0.001$ \\
\hline Hypertension & $2634(29.49)$ & $6315(35.35)$ & $4970(27.82)$ & $<0.001$ & 0.004 & $<0.001$ \\
\hline Dyslipidemia & 1095 (12.26) & 3640 (20.38) & $3211(17.98)$ & $<0.001$ & $<0.001$ & $<0.001$ \\
\hline Cardiovascular events & $227(2.54)$ & $729(4.08)$ & $480(2.69)$ & $<0.001$ & 0.48 & $<0.001$ \\
\hline Stroke & $299(3.35)$ & $944(5.28)$ & $878(4.92)$ & $<0.001$ & $<0.001$ & 0.11 \\
\hline
\end{tabular}

$\mathrm{HCV}=$ hepatitis $\mathrm{C}$ virus.

Values are $n(\%)$ or mean \pm standard deviation.

the development of schizophrenia. Previous findings that patients with schizophrenia had a higher prevalence of chronic liver disease than the general population, and comorbidity with diabetes was the primary risk factor for patients with schizophrenia to develop chronic liver disease, $, 9,27$ endorse a causal relationship between HCV infection and schizophrenia, because HCV infection is a main cause of both chronic liver disease and diabetes. ${ }^{1}$ Although schizophrenia is a psychiatric disorder with high heritability, the etiology of schizophrenia is complex and involves a complicated interplay of genetic and environmental factors. ${ }^{28}$ Infection, obstetric complications, urbanicity, cannabis and trauma have all been shown to interact with genetic risk. ${ }^{29}$ With respect to infection, associations between schizophrenia and toxoplasmosis, herpes or influenza have been reported, ${ }^{20-24,28}$ and we eliminated patients with these infections to avoid any confounding effects. Interestingly, $8 \%$ of the human genome consists of human endogenous retroviruses (HERVs) and a specific HERV - HERV-W - has been linked with schizophrenia. ${ }^{28}$ A lifelong scenario of a detrimental interaction between infectious agents and HERV-W genes may cause the development of schizophrenia. ${ }^{29}$ The evidence of direct neuroinvasion of $\mathrm{HCV}$, particularly in cells of the macrophage or monocyte lineage, ${ }^{30}$ suggest the possibility that HCV interacts with HERV-W in initiating the development of schizophrenia. As well, inflammation, oxidative stress ${ }^{31}$ and disturbances in neurotransmitter, metabolism, immunity and adipokine homeostasis ${ }^{32}$ may play important roles in eliciting schizophrenia. HCV infection consistently causes significant alterations in metabolism, ${ }^{1,33,34}$ neurotransmitter, ${ }^{34}$ immunity, adipokine ${ }^{15}$ and oxidative stress profiles ${ }^{1,33,34}$ in the hosts, reflecting the potential link between HCV and schizophrenia. It is important to note that the typical age of onset for schizophrenia is late adolescence or the early $20 s^{,}{ }^{6}$ 


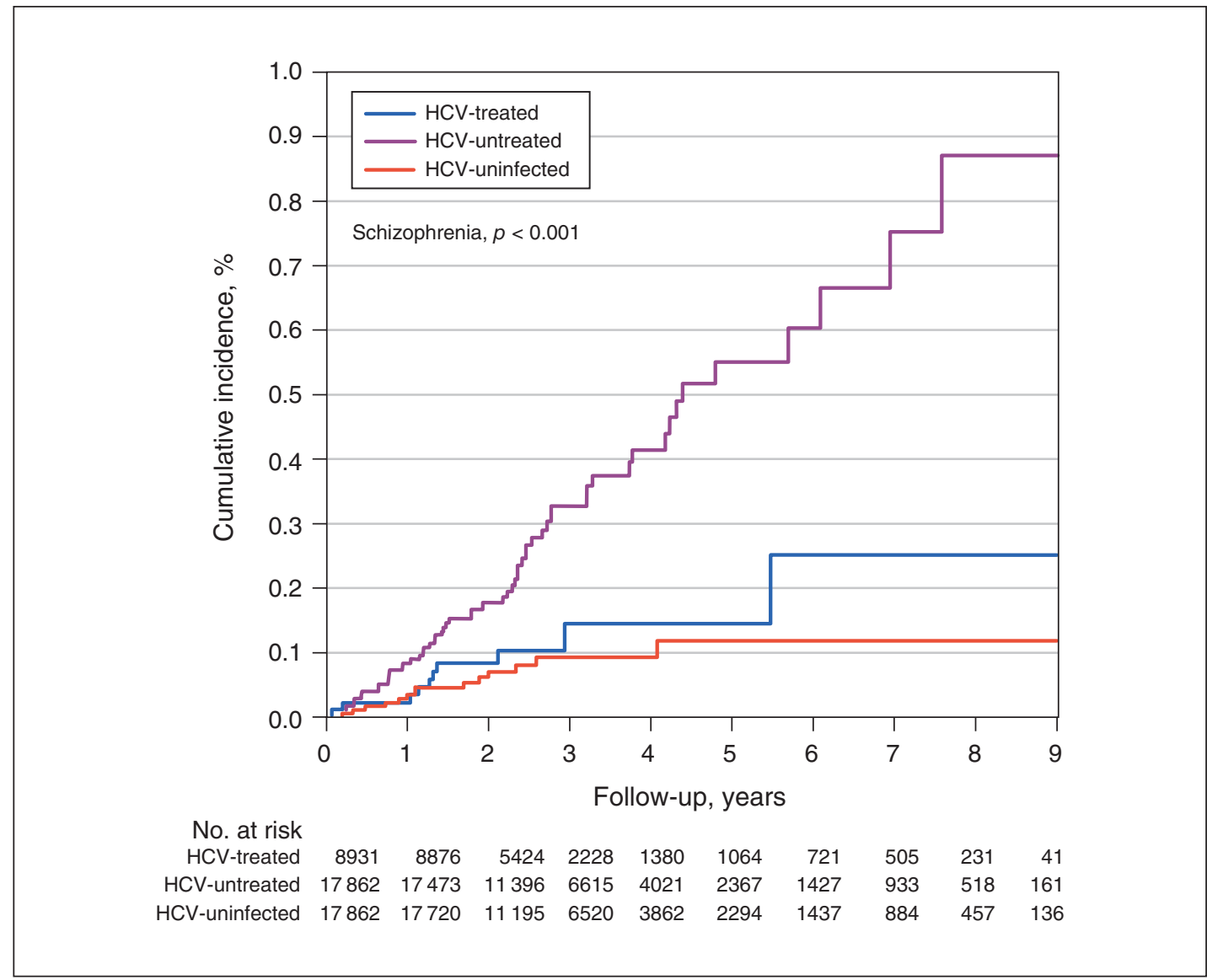

Figure 2: Cumulative incidence of schizophrenia among the 3 Taiwan National Health Insurance Research Database cohorts: HCV-treated, HCV-untreated and HCV-uninfected. HCV = hepatitis C virus.

Table 2: Comparison of the cumulative incidence of schizophrenia among the study cohorts

\begin{tabular}{|c|c|c|c|c|c|c|}
\hline \multirow[b]{2}{*}{ Characteristic } & \multirow[b]{2}{*}{ HCV-treated } & \multirow[b]{2}{*}{ HCV-untreated } & \multirow[b]{2}{*}{ HCV-uninfected } & \multicolumn{3}{|c|}{$p$ value } \\
\hline & & & & $\begin{array}{l}\text { HCV-treated } \\
\text { v. HCV- } \\
\text { untreated }\end{array}$ & $\begin{array}{l}\text { HCV-treated } \\
\text { v. HCV- } \\
\text { uninfected }\end{array}$ & $\begin{array}{c}\text { HCV-untreated } \\
\text { v. HCV- } \\
\text { uninfected }\end{array}$ \\
\hline Participants, $n$ & 8931 & 17862 & 17862 & - & - & - \\
\hline Years of follow-up, mean $\pm S D$ & $2.76 \pm 1.75$ & $2.98 \pm 1.84$ & $2.96 \pm 1.80$ & - & - & - \\
\hline Schizophrenia events, $n(\%)$ & $10(0.11)$ & $57(0.32)$ & $14(0.08)$ & - & - & - \\
\hline Competing mortality, $n(\%)$ & 207 (2.32) & $1283(7.18)$ & $389(2.18)$ & - & - & - \\
\hline $\begin{array}{l}\text { Cumulative incidence of } \\
\text { schizophrenia, \% }(95 \% \mathrm{Cl})\end{array}$ & $0.251(0.091-0.599)$ & $0.870(0.556-1.311)$ & $0.118(0.062-0.213)$ & 0.005 & 0.33 & $<0.001$ \\
\hline
\end{tabular}

whereas most of the patients in our study (82.81\%) were aged 40 years or older. That is, most of the diagnoses of schizophrenia in the present study occurred after age 40 years, because only those with no schizophrenia-related claims at least 2 years before baseline were enrolled. This finding was in line with the fact that the HCV infection rate is highest for adults older than 35 years, ${ }^{35}$ because the present study was based on propensity score-matched HCV-positive and HCV-negative cohorts. The late onset of schizophrenia in these patients thus might not be generalizable for patients with schizophrenia and requires further investigation.

Although interferon-based anti-HCV therapy in patients with schizophrenia has yielded negligible worsening psychiatric symptoms and the therapeutic response rate for people with schizophrenia is similar to that of the general population, ${ }^{36}$ schizophrenia is still a predictive factor for not treating 


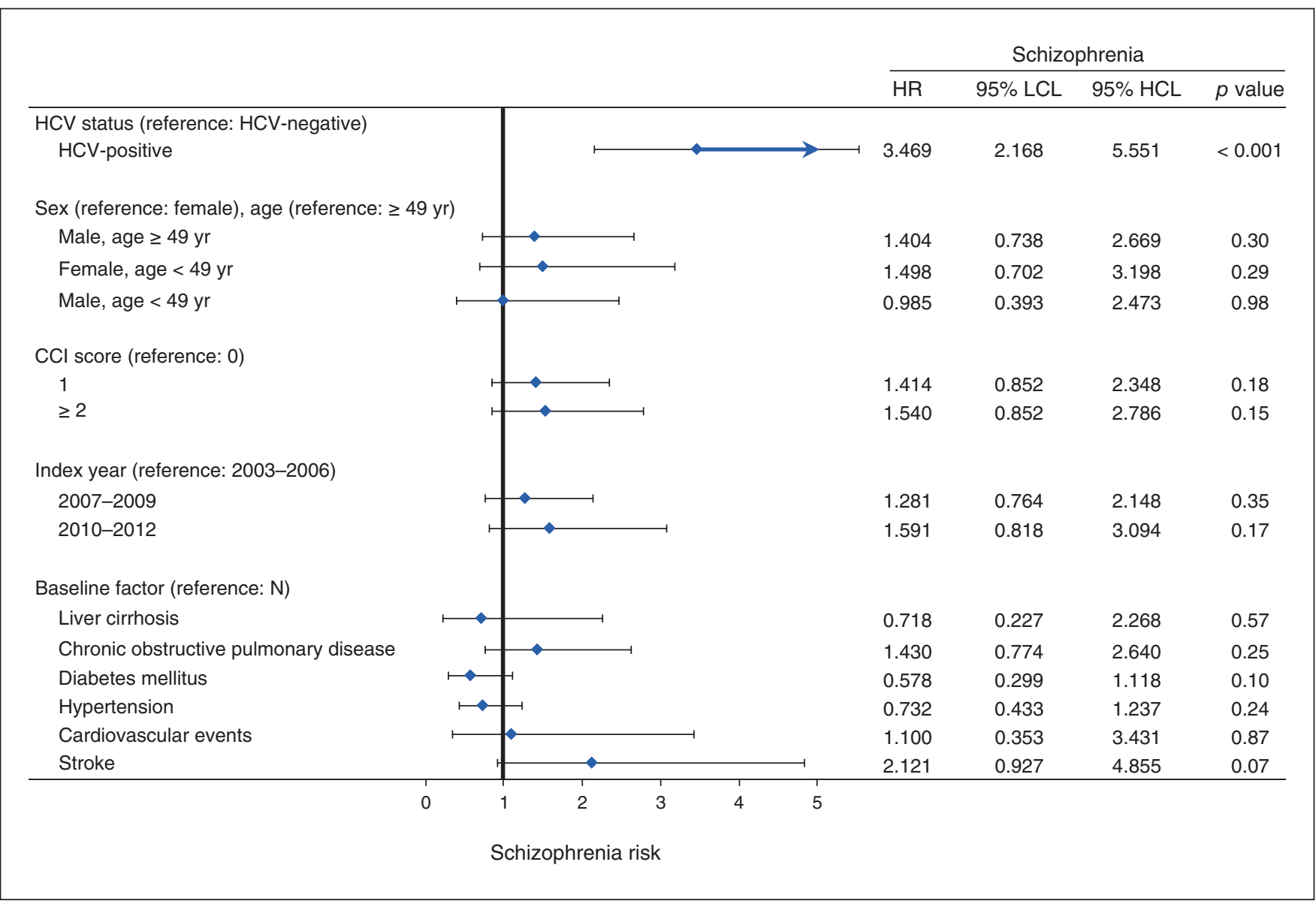

Figure 3: Forest plot of factors associated with incident schizophrenia in the 2 Taiwan National Health Insurance Research Database cohorts: HCV-positive (HCV-untreated) and HCV-negative (combination of HCV-treated and HCV-uninfected). CCI = Charlson Comorbidity Index score; $\mathrm{HCL}=$ higher confidence interval limit; $\mathrm{HCV}=$ hepatitis $\mathrm{C}$ virus; $\mathrm{HR}=$ hazard ratio; $\mathrm{LCL}=$ lower confidence interval limit.

HCV infection because of concerns about interferon-related neuropsychiatric adverse effects. ${ }^{37}$ We stress that the HCVassociated risk in incident schizophrenia might be reversed by interferon-based anti-HCV therapy, because the cumulative incidence of schizophrenia in the HCV-treated cohort was lower than that of the HCV-untreated cohort and similar to that of the HCV-uninfected cohort. However, whether the reversal of $\mathrm{HCV}$-associated risk in schizophrenia is universal for all anti-HCV regimens (including DAAs) or is specific to interferon-based therapy requires further investigation. Moreover, the HCV-untreated cohort in the present study had the highest risk of overall mortality among the 3 cohorts. It is likely that this finding was at least partly attributable to $\mathrm{HCV}$-associated systemic complications. ${ }^{1-4}$ As well, people with schizophrenia are at greater risk for morbidity and mortality as a result of cardiovascular disease ${ }^{38}$ and stroke ${ }^{39}$ than the general population. Thus, mortality is high for those with schizophrenia and HCV infection. ${ }^{40}$ These findings point to the importance of prescribing anti-HCV therapy in all HCVinfected patients regardless of schizophrenia status (unless contraindicated), particularly in the era of DAAs, which can cure $\mathrm{HCV}$ infection. In addition to $\mathrm{HCV}$ infection, stroke was borderline positively associated with the development of incident schizophrenia. Schizophrenia-like psychosis after stroke is common and severely affects quality of life. ${ }^{41}$ Preexisting subcortical atrophy and a lesion location in the right hemisphere ${ }^{42}$ - particularly in the frontal, temporal and parietal regions and the right caudate nucleus ${ }^{41}$ — are risk factors for post-stroke schizophrenia-like psychosis.

\section{Limitations}

The current study had several limitations. First, because the TNHIRD did not contain direct laboratory results, it was impossible to explore a correlation between schizophrenia and SVR to anti-HCV therapy. However, we were confident of antiviral efficacy in the HCV-treated cohort because interferon-based therapy generally achieves a high SVR in Taiwan (up to $82.7 \%$ ), ${ }^{15}$ where a favourable IFNL3 genetic variation for interferon-based therapy response is prevalent. ${ }^{15}$ Second, there might have been some patients with undiagnosed HCV infection in the HCV-uninfected cohort. However, given that reimbursement for anti-HCV therapy is nationwide and only $2.7 \%$ of the Taiwanese were likely to be 
infected with $\mathrm{HCV},{ }^{43}$ the rate of undiagnosed $\mathrm{HCV}$ infection in the HCV-uninfected cohort was probably negligible and likely would not bias our results. Third, information about important risk factors for schizophrenia, including genetic susceptibility, were lacking in the TNHIRD and the effect of these risk factors on schizophrenia could not be assessed. Fourth, we were unable to determine the precise mechanism of the increased risk of schizophrenia in patients with $\mathrm{HCV}$ infection. Future prospective studies in other independent cohorts with identifiable SVR following DAA-based anti-HCV therapy, comprehensive surveys of risk factors, well-stratified patient subpopulations and sophisticated molecular or animal investigations are needed to elucidate the fundamental mechanisms that underlie the findings described here.

\section{Conclusion}

Taken together, the findings of the present study confirmed that in a population aged mostly 40 years and older, HCV positivity is a positive risk factor for incident schizophrenia, and that the risk may be reversed with interferon-based antiviral therapy. In the era of DAAs to eliminate HCV infection, anti-HCV therapy should be prescribed for all people infected with HCV to reduce the risk not only of hepatic complications, but also of extrahepatic complications, including schizophrenia.

Acknowledgments: The authors thank Ms. Shu-Chun Chen from the Liver Research Center, Chang Gung Memorial Hospital, Taiwan, for assistance with data mining.

Affiliations: From the Clinical Informatics and Medical Statistics Research Center, College of Medicine, Chang Gung University, Taiwan (Cheng, Ku); the Department of Emergency Medicine, Chang Gung Memorial Hospital, Keelung, Taiwan (Cheng); the Department of Gastroenterology and Hepatology, Department of Internal Medicine, Chang Gung Memorial Hospital, Yunlin, Taiwan (Hu); the Division of Pediatric Neurologic Medicine, Chang Gung Children's Hospital, Taoyuan, Taiwan (M.-Y. Chang); the Division of Pediatric General Medicine, Chang Gung Children's Hospital, Taoyuan, Taiwan (M.-Y. Chang); the Department of Cardiology, Heart Failure Center, Chang Gung Memorial Hospital, Taiwan (Lin); the Department of Cardiology, Chang Gung Memorial Hospital, Taoyuan, Taiwan (Lin); the Liver Research Center, Division of Hepatology, Department of Gastroenterology and Hepatology, Chang Gung Memorial Hospital, Taoyuan, Taiwan (Chien, M.-L. Chang); and the Department of Medicine, College of Medicine, Chang Gung University, Taoyuan, Taiwan (Chien, M.-L. Chang).

Funding: This study was supported by grants from the Chang Gung Medical Research Program (CMRPG3I0412, CMRPG3K0721 and CMRPG1K0111) and the National Science Council (MOST 110-2314B-182-044-, MOST 109-2314-B-182-024- and MOST 109-2629-B-182002-). The funders had no role in study design, data collection and analysis, decision to publish, or preparation of the manuscript. The opinions expressed in this paper are those of the authors and do not necessarily represent those of Chang Gung Medical Hospital and National Science Council, Taiwan.

Competing interests: None declared.

Contributors: M. Lin and M.-L. Chang designed the study. J. Hu and R. Chien acquired the data, which J. Cheng, M.-Y. Chang and H. Ku analyzed. All authors wrote the article. All authors approved the final version to be published and can certify that no other individuals not listed as authors have made substantial contributions to the paper.
Content licence: This is an Open Access article distributed in accordance with the terms of the Creative Commons Attribution (CC BY-NC-ND 4.0) licence, which permits use, distribution and reproduction in any medium, provided that the original publication is properly cited, the use is noncommercial (i.e., research or educational use), and no modifications or adaptations are made. See: https:// creativecommons.org/licenses/by-nc-nd/4.0/

\section{References}

1. Chang ML. Metabolic alterations and hepatitis C: from bench to bedside. World J Gastroenterol 2016;22:1461-76.

2. Mohd Hanafiah K, Groeger J, Flaxman AD, et al. Global epidemiology of hepatitis $C$ virus infection: new estimates of age-specific antibody to HCV seroprevalence. Hepatology 2013;57:1333-42.

3. Ramos-Casals M, Stone JH, Cid MC, et al. The cryoglobulinaemias. Lancet 2012;379:348-60.

4. Adinolfi LE, Nevola R, Lus G, et al. Chronic hepatitis C virus infection and neurological and psychiatric disorders: an overview. World J Gastroenterol 2015;21:2269-80.

5. Molinaro L, Hui P, Tan M, Mishra RK. Role of presynaptic phosphoprotein synapsin II in schizophrenia. World J Psychiatry 2015;5: 260-72.

6. Asor E, Ben-Shachar D. Platelets: a possible glance into brain biological processes in schizophrenia. World J Psychiatry 2012;2: 124-33.

7. Vawter MP, Mamdani F, Macciardi F. An integrative functional genomics approach for discovering biomarkers in schizophrenia. Brief Funct Genomics 2011;10:387-99.

8. Weickert CS, Rothmond DA, Purves-Tyson TD. Considerations for optimal use of postmortem human brains for molecular psychiatry: lessons from schizophrenia. Handb Clin Neurol 2018;150:221-35.

9. Fuller BE, Rodriguez VL, Linke A, et al. Prevalence of liver disease in veterans with bipolar disorder or schizophrenia. Gen Hosp Psychiatry 2011;33:232-7.

10. Nakamura Y, Koh M, Miyoshi E, et al. High prevalence of the hepatitis $C$ virus infection among the inpatients of schizophrenia and psychoactive substance abuse in Japan. Prog Neuropsychopharmacol Biol Psychiatry 2004;28:591-7.

11. Himelhoch S, McCarthy JF, Ganoczy D, et al. Understanding associations between serious mental illness and hepatitis $C$ virus among veterans: a national multivariate analysis. Psychosomatics 2009; 50:30-7.

12. Freudenreich O, Gandhi RT, Walsh JP, et al. Hepatitis C in schizophrenia: screening experience in a community-dwelling clozapine cohort. Psychosomatics 2007;48:405-11.

13. Hung CC, Loh EW, Hu TM, et al. Prevalence of hepatitis B and hepatitis $C$ in patients with chronic schizophrenia living in institutions. J Chin Med Assoc 2012;75:275-80.

14. Chiu YL, Lin HC, Kao NW, et al. Increased risk of concurrent hepatitis C among male patients with schizophrenia. Psychiatry Res 2017; 258:217-20.

15. Chang ML, Hsu CM, Lin $\mathrm{CH}$, et al. The evolving interplay among abundant adipokines in patients with hepatitis $\mathrm{C}$ during viral clearance. Nutrients 2017;9:E570.

16. D'Ambrosio R, Pasulo L, Puoti M, et al. Real-world effectiveness and safety of glecaprevir/pibrentasvir in 723 patients with chronic hepatitis C. J Hepatol 2019;70:379-87.

17. Toyoda H, Kumada T, Tada T, et al. Risk factors of hepatocellular carcinoma development in non-cirrhotic patients with sustained virologic response for chronic hepatitis C virus infection. J Gastroenterol Hepatol 2015;30:1183-9.

18. Hu JH, Chen MY, Yeh CT, et al. Sexual dimorphic metabolic alterations in hepatitis $C$ virus-infected patients: a community-based study in a hepatitis $\mathrm{B} /$ hepatitis $\mathrm{C}$ virus hyperendemic area. Medicine (Baltimore) 2016;95:e3546.

19. Chang ML, Lin YJ, Chang CJ, et al. Occult and overt HBV coinfections independently predict postoperative prognosis in HCVassociated hepatocellular carcinoma. PLoS One 2013;8:e64891. 
20. Tomasik J, Schultz TL, Kluge W, et al. Shared immune and repair markers during experimental toxoplasma chronic brain infection and schizophrenia. Schizophr Bull 2016;42:386-95.

21. Melamede R. Parasitic brain infection, endocannabinoids, and schizophrenia. Med Hypotheses 2009;72:220-2.

22. Contini C, Seraceni S, Cultrera R, et al. Chlamydophila pneumoniae infection and its role in neurological disorders. Interdiscip Perspect Infect Dis 2010;2010:273573.

23. Bonthius DJ, Perlman S. Congenital viral infections of the brain: lessons learned from lymphocytic choriomeningitis virus in the neonatal rat. PLoS Pathog 2007;3:e149.

24. Engel JA, Zhang J, Bergström T, et al. Neonatal herpes simplex virus type 1 brain infection affects the development of sensorimotor gating in rats. Brain Res 2000;863:233-40.

25. Deyo RA, Cherkin DC, Ciol MA. Adapting a clinical comorbidity index for use with ICD-9-CM administrative databases. J Clin Epidemiol 1992;45:613-9.

26. Gray RJ. A class of K-sample tests for comparing the cumulative incidence of a competing risk. Ann Stat 1998;16:1141-54.

27. Hsu JH, Chien IC, Lin CH, et al. Increased risk of chronic liver disease in patients with schizophrenia: a population-based cohort study. Psychosomatics 2014;55:163-71.

28. Leboyer M, Tamouza R, Charron D, et al. Human endogenous retrovirus type $\mathrm{W}(\mathrm{HERV}-\mathrm{W})$ in schizophrenia: a new avenue of research at the gene-environment interface. World J Biol Psychiatry 2013;14:80-90.

29. Réthelyi JM, Benkovits J, Bitter I. Genes and environments in schizophrenia: the different pieces of a manifold puzzle. Neurosci Biobehav Rev 2013;37:2424-37.

30. Radkowski M, Wilkinson J, Nowicki M, et al. Search for hepatitis $C$ virus negative-strand RNA sequences and analysis of viral sequences in the central nervous system: evidence of replication. J Virol 2002;76:600-8.

31. Mitra S, Natarajan R, Ziedonis D, et al. Antioxidant and antiinflammatory nutrient status, supplementation, and mechanisms in patients with schizophrenia. Prog Neuropsychopharmacol Biol Psychiatry 2017;78:1-11.
32. Harris LW, Guest PC, Wayland MT, et al. Schizophrenia: metabolic aspects of aetiology, diagnosis and future treatment strategies. Psychoneuroendocrinology 2013;38:752-66.

33. Chang SW, Cheng ML, Shiao MS, et al. Recovery of lipid metabolic alterations in hepatitis $C$ patients after viral clearance: incomplete restoration with accelerated $\omega$-oxidation. J Clin Lipidol 2018;12: 756-66.

34. Chang ML, Cheng ML, Chang SW, et al. Recovery of pangenotypic and genotype-specific amino acid alterations in chronic hepatitis $C$ after viral clearance: transition at the crossroad of metabolism and immunity. Amino Acids 2017;49:291-302.

35. Posuwan N, Wanlapakorn N, Sintusek P, et al. Towards the elimination of viral hepatitis in Thailand by the year 2030. J Virus Erad 2020; 6:100003.

36. Huckans M, Mitchell A, Pavawalla S, et al. The influence of antiviral therapy on psychiatric symptoms among patients with hepatitis C and schizophrenia. Antivir Ther 2010;15:111-9.

37. Butt AA, Justice AC, Skanderson M, et al. Rate and predictors of treatment prescription for hepatitis C. Gut 2007;56:385-9.

38. Curkendall SM, Mo J, Glasser DB, et al. Cardiovascular disease in patients with schizophrenia in Saskatchewan, Canada. J Clin Psychiatry 2004;65:715-20.

39. Tsai KY, Lee CC, Chou YM, et al. The incidence and relative risk of stroke in patients with schizophrenia: a five-year follow-up study. Schizophr Res 2012;138:41-7.

40. Gimelfarb Y, Becalel E, Wolf A, et al. Impact of chronic hepatitis C virus (HCV) on survival of schizophrenic patients with co-occurring substance use disorders followed for ten years. Harefuah 2014; 153:641-5, 688.

41. Stangeland H, Orgeta V, Bell V. Poststroke psychosis: a systematic review. J Neurol Neurosurg Psychiatry 2018;89:879-85.

42. Rabins PV, Starkstein SE, Robinson RG. Risk factors for developing atypical (schizophreniform) psychosis following stroke. J Neuropsychiatry Clin Neurosci 1991;3:6-9.

43. Cheng YL, Wang YC, Lan KH et al. Anti-hepatitis C virus seropositivity is not associated with metabolic syndrome irrespective of age, gender and fibrosis. Ann Hepatol 2015;14:181-9. 